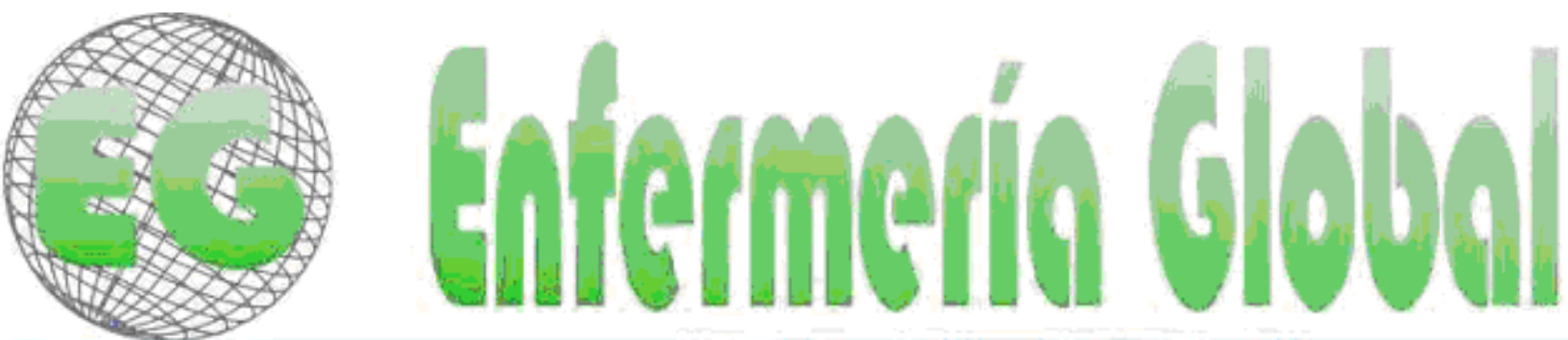

15SN 1696-6*5:

Prevista electrônica cuatrimestral de Enfermeria

$\mathrm{N}^{\circ} 20$

Octubre 2010

www.um.es/egloball

REVISIONES

\title{
PRODUCCIÓN CIENTÍFICA EN ENFERMERÍA DE URGENCIAS CARDÍACAS Y CORONARIAS. ANÁLISIS DE LA LITERATURA.
}

SCIENTIFIC PRODUCTION ON CARDIAC AND CORONARY EMERGENCY NURSING: LITERATURE ANALYSIS

\author{
*Castro Teijeiro, Jesús \\ *Enfermero. Complexo Hospitalario Xeral-Calde de Lugo. \\ Palabras clave: Enfermería, Urgencias, Cardiología. \\ Keywords: Emergencies, Nursing, and Cardiology.
}

\section{RESUMEN}

Objetivo principal: conocer cuánto y cómo se está publicando en el ámbito de la enfermería de urgencias cardíacas y coronarias, y cómo se recupera esa literatura.

Metodología: la búsqueda bibliográfica se hizo en Medline y Cinahl, utilizando como palabras clave "Emergency Nursing"[Mesh] AND "Heart Diseases"[Mesh]; en Cuiden y en el IME, utilizando como palabras clave "Enferm*" "Card*" y "Urgen*". Se ha limitado la búsqueda a los artículos publicados entre 2003 y 2008, ambos incluidos. Las variables analizadas fueron: año de publicación, idioma del artículo, filiación laboral y ámbito geográfico del autor firmante, revista en la que fue publicado y tipo de artículo.

Resultados principales: se han encontrado 181 artículos en Medline y Cinahl, en las bases de datos nacionales no se obtuvo ningún resultado. El 28 '18\% fueron publicados en 2008 ; el 95 '18\% han sido escritos en inglés; el $44{ }^{\prime} 20 \%$ fueron escritos por autores estadounidenses, no se ha encontrado ninguno procedente de España.

Conclusión principal: la producción científica en enfermería de urgencias cardíacas y coronarias es escasa. La recuperación presenta deficiencias debido a que las bases de datos no muestran algunas informaciones sobre el artículo (autor, tipo de artículo, tema).

\section{ABSTRACT}

Main Objective: To know how many publications have been produced on the subject of cardiac and coronary emergency nursing field and the way they have been written, and discover how this literature can be recovered

Methodology: The bibliographic review has been done through Medline and Cinahl, by using keywords as "Emergency Nursing" [Mesh] AND "Heart Disease" [Mesh]; Cuiden and IME, by using key words as "Enferm*", "Card" and "Urgen". The search was carried out between 2003 and 2008 inclusive. The analyzed variables were: publication date, the language used in the article, the author's 
work affiliation and geographic area, the magazine in which the article was published and type of article.

Main results: 181 articles have been found in Medline and Cinahl, no results have been obtained from national database. $28.18 \%$ of them were published in 2008, 95.18\% were written in English, $44.20 \%$ were written by American authors. None of them were Spanish.

Main Conclusion: There is poor scientific production on cardiac and coronary emergency nursing. It is hard to get all of the information due to a lack of information in the database such as author, article type or theme.

\section{INTRODUCCIÓN}

Si bien el origen de la investigación de la Enfermería está datado en 1854 con la observación de Florence Nightingale sobre los efectos de las acciones enfermeras (1), esta no ha sido reconocida plenamente, a pesar de que ha hecho un progreso notable en las últimas décadas. Frente al tradicional sistema de investigación basado en reglas, principios y tradiciones que se transmitían por el método de aprendizaje artesanal y por la sabiduría común que se obtiene con los años de experiencia, desde los años cincuenta la enfermería inició esfuerzos por desarrollar nuevos sistemas.

A pesar de este esfuerzo, la investigación en enfermería es todavía una empresa pequeña. El volumen de publicación es escaso, lo cual limita el conocimiento de los hallazgos y por lo tanto la aplicación de estos a la práctica. Así, una de las principales necesidades de la enfermería en nuestros días es hacer investigación capaz de mejorar los fundamentos para la práctica, y de gran importancia conocer el estado actual de la investigación para dar el gran paso y consolidar la profesión de enfermería. Por esta razón han surgido numerosos estudios que han tratado de analizar la investigación y producción científica de la enfermería desde diferentes aspectos, como es el caso de Díaz Benavente y Martín Leal (2), y Paluci Marziale, Costa Mendes (3) y Jiménez Hernandez (4).

En lo que respecta a la enfermería de urgencias, se han escrito artículos que también analizan la producción científica, como Alpi (5), que analizó una serie de revistas clave en el ámbito de la enfermería de urgencias de acuerdo a las citaciones recibidas o la cobertura de estas en las bases de datos. En el ámbito nacional Navalpotro estudió el estado de la investigación en España, prioridades tendencias futuras, financiación... (6).

\section{OBJETIVO}

Este estudio evalúa las publicaciones en enfermería de urgencias cardíacas y coronarias entre 2003 y 2008 utilizando diferentes indicadores bibliométricos, con el fin de conocer de qué manera se está publicando en este ámbito y analizar cuáles son los temas más recurridos.

Como objetivo secundario se ha planteado la forma en que se recupera la información por la base de datos, si ofrece la información suficiente sobre un artículo para poder emplearlo.

\section{METODOLOGÍA}

El artículo se refiere a la producción científica en Enfermería de situaciones de urgencia por patología cardíaca o coronaria a nivel internacional.

Se trata de un estudio descriptivo transversal. Para su realización se llevaron a cabo los siguientes pasos: 
Búsqueda bibliográfica en bases de datos tanto de ámbito internacional: Medline, la principal base de datos de la National Library of Medicine y Cinahl (Cumulative Index to Nursing and Allied Health Literature), como nacional: Cuiden, una de las principales bases de datos en España, es elaborada por la Fundación Index; e IME, elaborada por el CSIC (Consejo Superior de Investigaciones Científicas), que recoge la producción biosanitaria española..

La estrategia de búsqueda empleada en Medline y Cinahl ha sido utilizando como palabras clave "Emergency Nursing"[Mesh] AND "Heart Diseases"[Mesh]. En las bases de datos nacionales la estrategia que se ha seguido ha sido combinando los términos "Enferm" "Card*" y "Urgen*" con el operador boleano AND. La única limitación que se ha puesto a los resultados ha sido que fuesen artículos publicados entre los años 2003 y 2008, ambos incluidos.

Gestión y eliminación de duplicados: Una vez realizada la búsqueda, los artículos obtenidos han sido descargados al gestor de referencias Endnote Web, con el fin de eliminar los posibles duplicados de las diferentes bases de datos.

Análisis de las variables. Para el análisis de variables se ha empleado el programa Microsoft Office Excel 2007, por ser compatible con el endnote web y permitir la introducción de diferentes variables de modo simultáneo y explotación de las mismas. Las variables seleccionadas han sido las que se presentan a continuación: año de publicación; idioma; autoría: ámbito laboral del autor firmante y lugar geográfico del autor firmante; tipo de artículo; revista en la que ha sido publicado y tema principal.

\section{RESULTADOS}

Se han obtenido 181 artículos una vez eliminados los duplicados de las bases de datos Medline y Cinahl. No se han encontrado resultados en las bases de datos nacionales.

Los datos obtenidos se van a exponer según las variables analizadas: año de publicación, idioma del artículo, país en el que se ha realizado el artículo.

Según el año de publicación, de los 181 artículos analizados se ha encontrado que el 13 ' $26 \%$ han sido publicados en el año 2003 ; el 12 '71\% en 2004 ; el 20 ' $99 \%$ en 2005 ; el 12 ' $15 \%$ en 2006; el 12'71\% en 2007; y el 28'18\% en 2008 (Figura 1). 


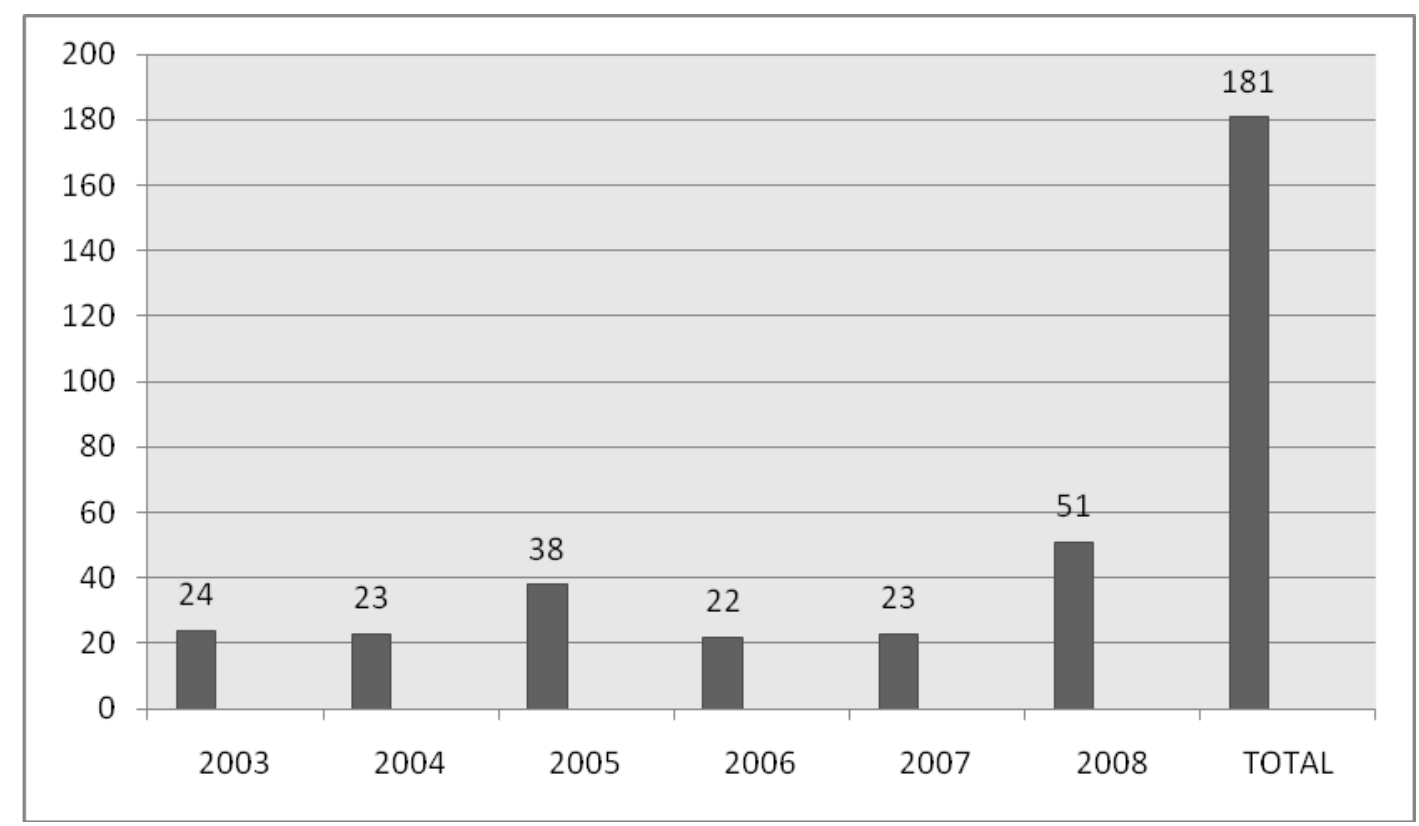

Figura 1. Número de artículos según año de publicación.

En cuanto al idioma en el que han sido publicados: el 95'58\% de los artículos están escritos en inglés, el $1{ }^{\prime} 66 \%$ en francés, 1 ' $66 \%$ en portugués, 0 ' $55 \%$ en italiano y 0 ' $55 \%$ en finés. No se ha encontrado ningún artículo en español (Figura 2).

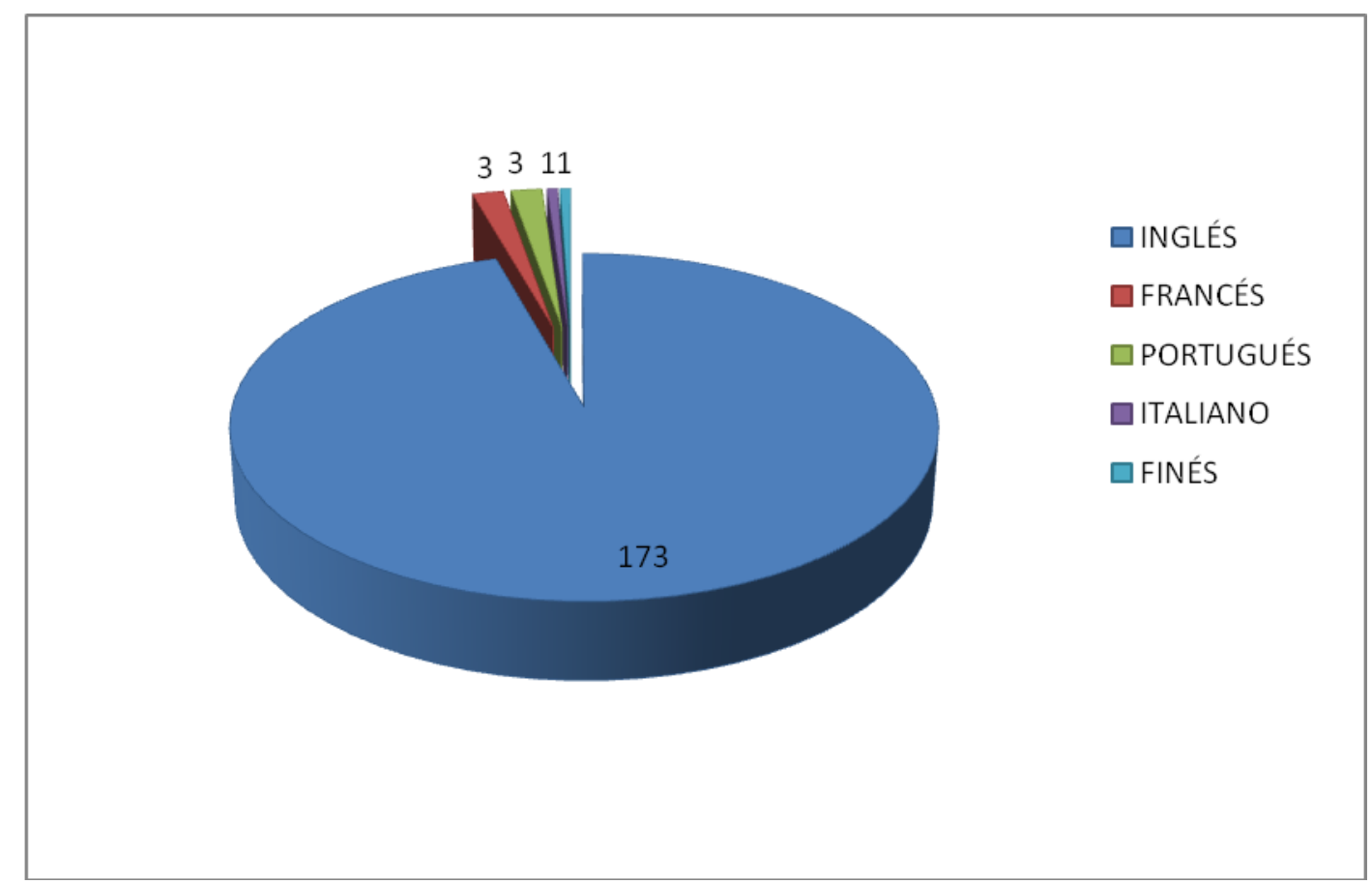

Figura 2. Número de artículos según idioma.

Según el país de procedencia del autor firmante, el $44^{\prime} 20 \%$ de los artículos han sido escritos por autores estadounidenses, el $16{ }^{\prime} 67 \%$ en el Reino Unido, el ${ }^{\prime} ' 66 \%$ en Brasil, el $1^{\prime} 66 \%$ en Canadá, el $1^{\prime} 66 \%$ en Irlanda, el $1{ }^{\prime} 10 \%$ en Australia, el $1{ }^{\prime} 10 \%$ en Finlandia, el $1{ }^{\prime} 10 \%$ en Italia, el $1{ }^{\prime} 10 \%$ en Nueva Zelanda, y hay un $29^{\prime} 83 \%$ de los artículos que no indican el país. No se ha localizado ninguna publicación procedente de España (Figura 3). 


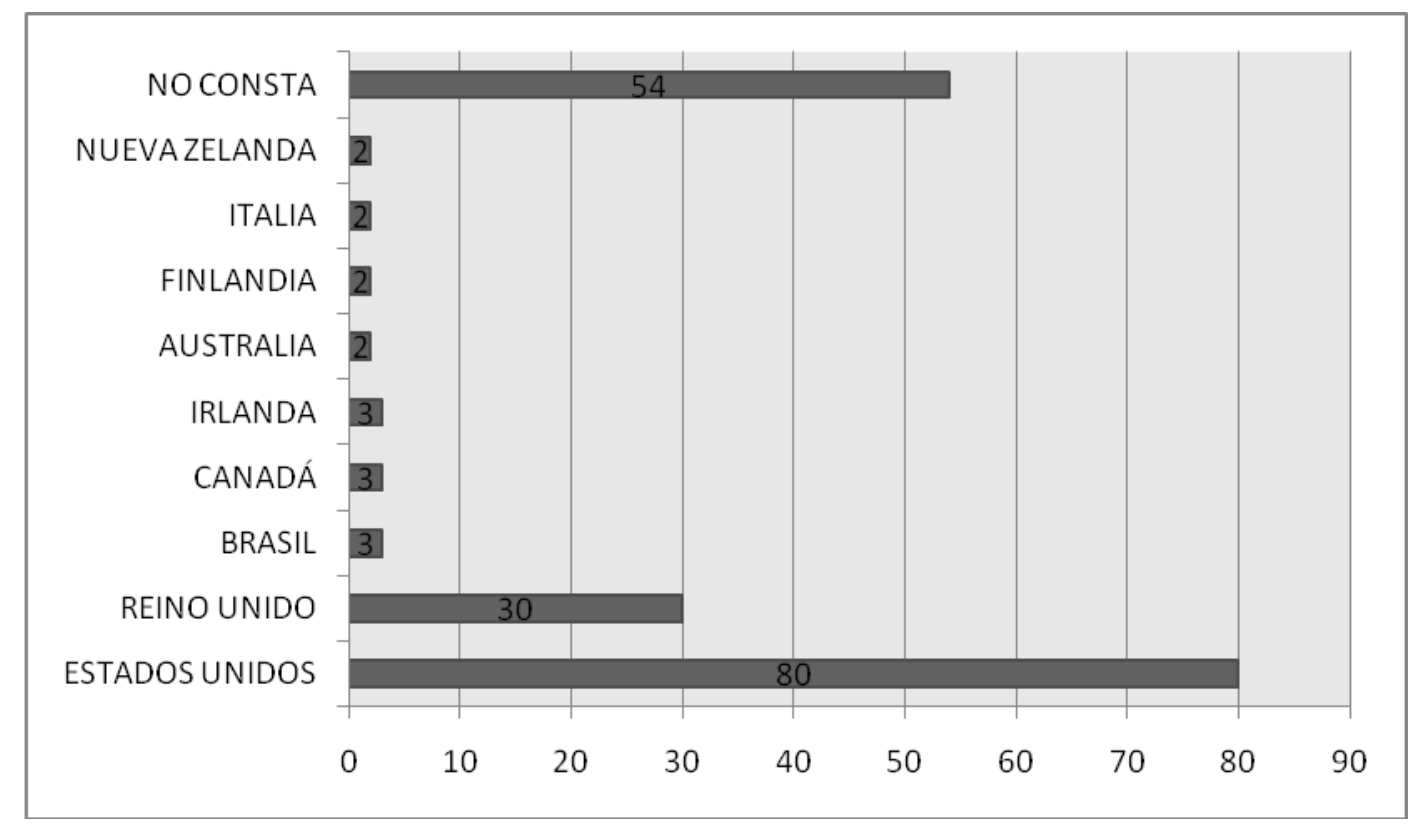

Figura 3. Número de artículos según ámbito geográfico del autor firmante.

En cuanto a la filiación laboral del autor, en un 30'94\% del total de artículos no se ha facilitado la información por la base de datos. En el resto de los casos analizados, se ha cuantificado un $40{ }^{\prime} 33 \%$ de artículos elaborados desde el ámbito hospitalario, un $28{ }^{\prime} 73 \%$ relacionados con el ámbito universitario (Figura 4). En un 20 '99\% no se ha encontrado el autor del artículo.

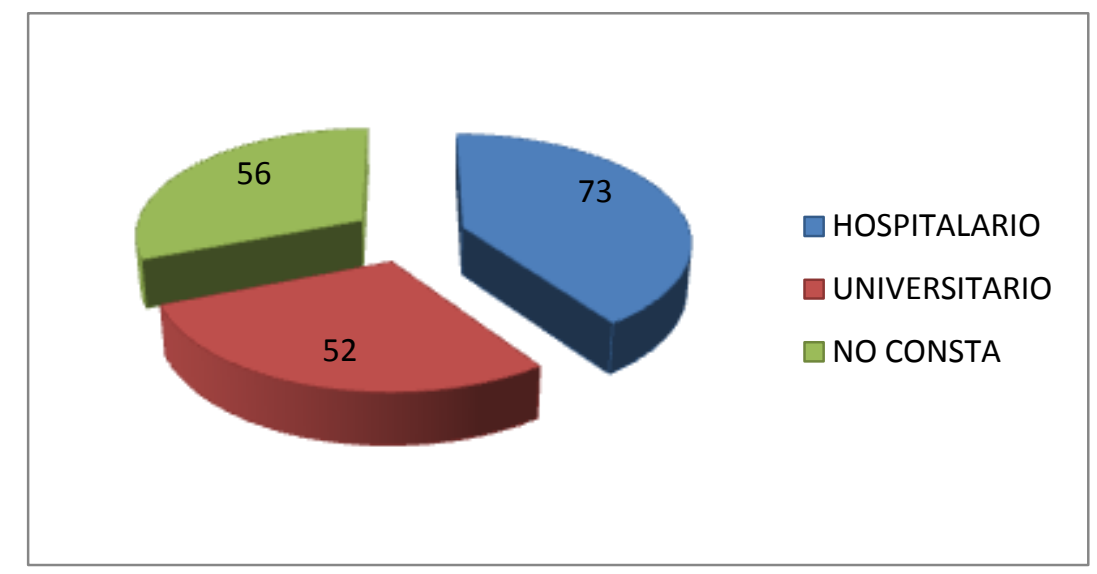

Figura 4. Número de artículos según ámbito profesional del autor firmante.

Hay un gran número de artículos sin tipología ofrecida por la base de datos, un 34 '25\% del total. Si se detallan los que más abundan, se encuentran: revisiones (14'36\%), informes de casos (9'39\%), estudios de casos (8'29\%), imágenes y gráficos $\left(6^{\prime} 63 \%\right)$, y revisiones e informe de casos (5’52\%). En la figura 5 se especifica el tipo de artículo. 


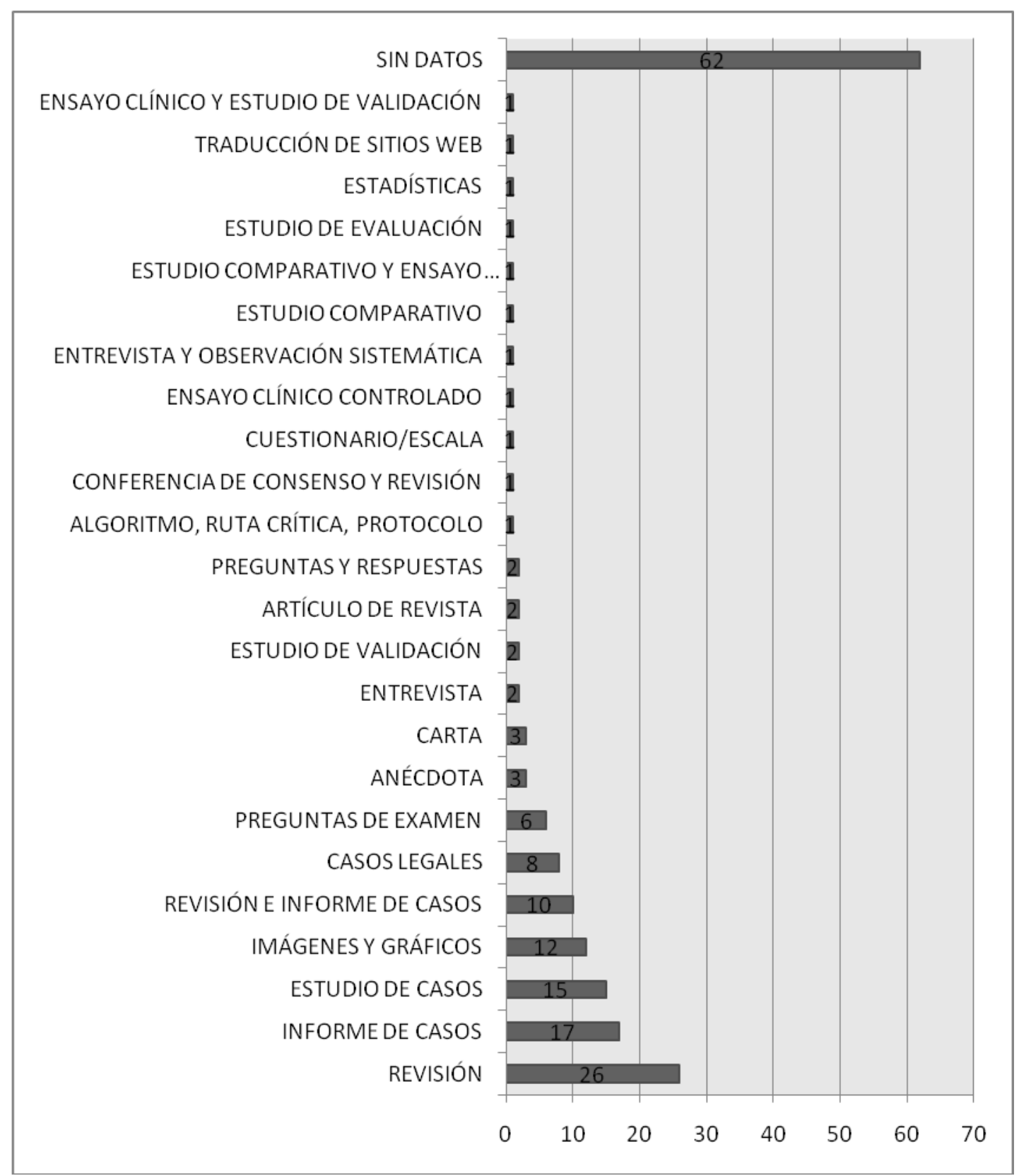

Figura 5. Número de artículos según tipología.

Un 92'26\% de los artículos han sido publicados en revistas de enfermería, de estos, el 56 '29\% están publicados en revistas de enfermería general, y el $43{ }^{\prime} 71 \%$ en revistas de enfermería especializada. Un 6'08\% han sido publicados en revistas médicas, y un 1'66\% en otros tipos de revista. Del porcentaje de artículos publicados en revistas de enfermería, se encuentra un $25^{\prime} 75 \%$ con temática médica (diagnóstico, tratamiento médico...).

Las revistas escogidas para la publicación de los artículos, son, la mayoría del ámbito de la enfermería. Las 5 revistas que cuentan con mayor número de artículos de enfermería en urgencias cardíacas y coronarias son: ED Nursing (20'44\%), Journal of Emergency Nursing (16'57\%), Emergency Nursing (7’73\%), Nursing (5’52\%), y Nursing Spectrum (con un 4'42\%) (Figura 6). 


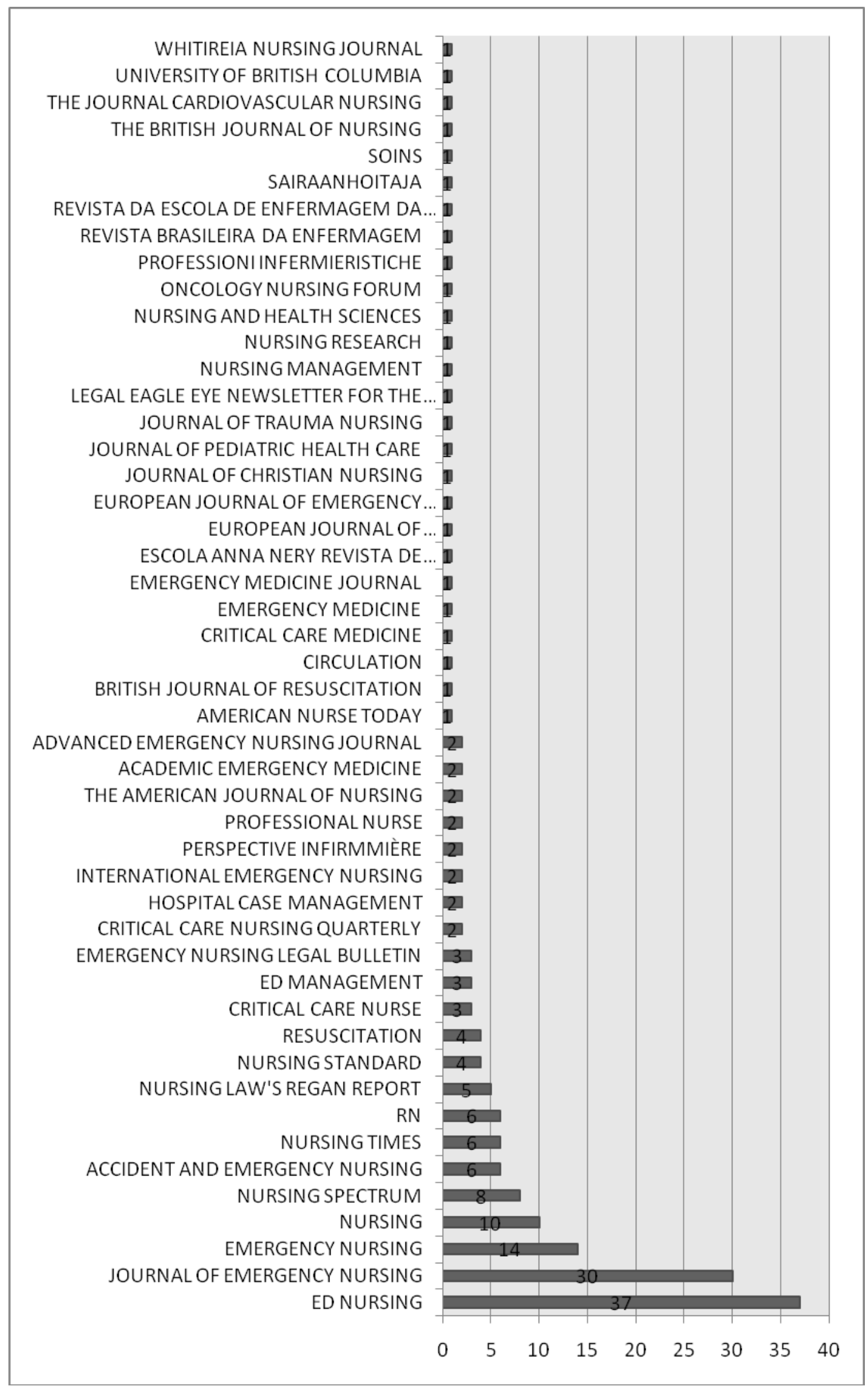

Figura 6. Número de artículos según revista en la que han sido publicados. 
La temática tratada en los artículos resulta muy variada y dispersa, siendo los temas más abundantes los relacionados con patologías concretas (coronariopatías, dolor torácico, infarto de miocardio, insuficiencia cardíaca, patología pericárdica, taponamiento cardíaco y taquicardia), relacionados con el tratamiento, con la electrocardiografía y con la atención en urgencias. Por otro lado, hay un $11{ }^{\prime} 60 \%$ del total de los artículos que no se han podido clasificar según su temática (Figura 7).

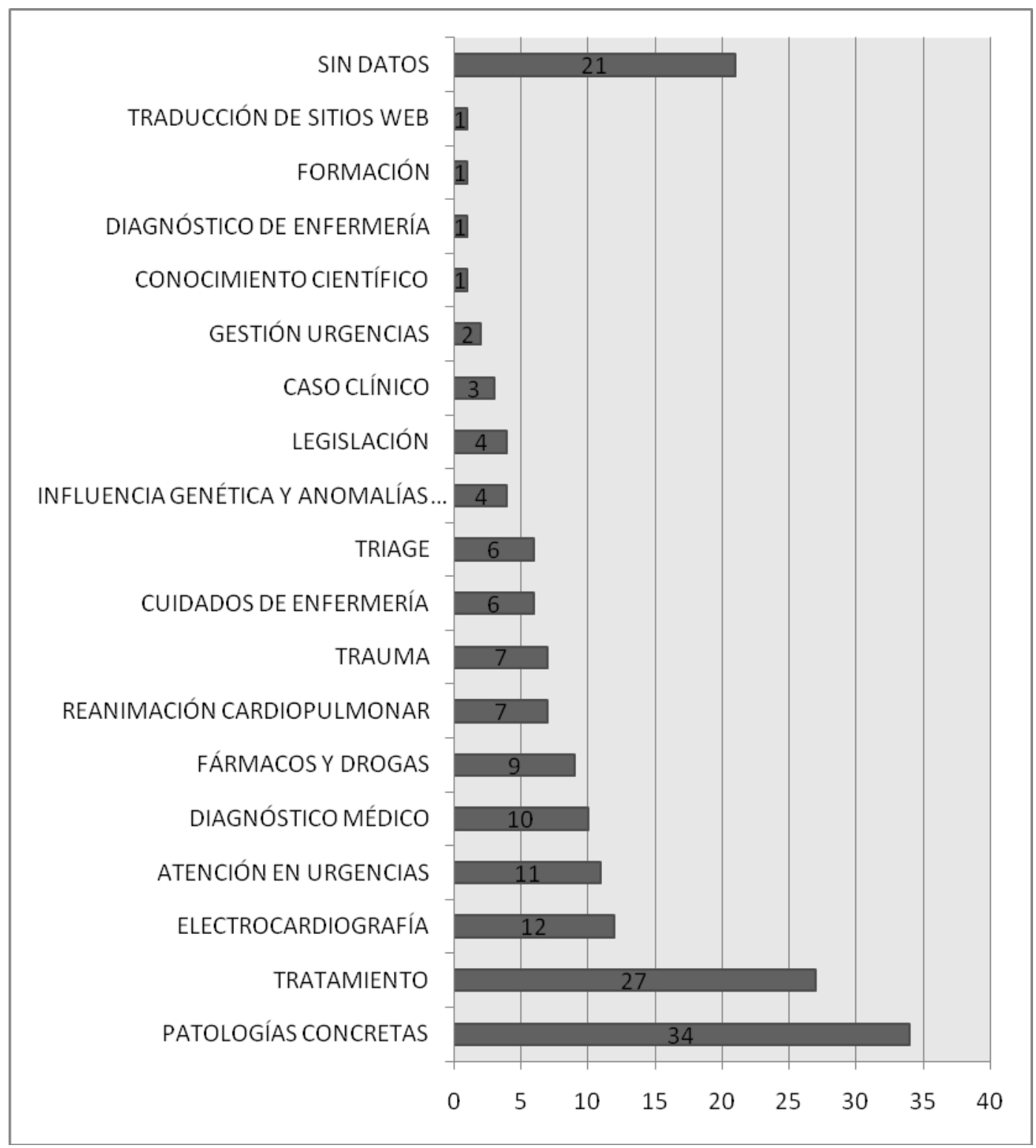

Figura 7. Número de artículos según tema principal.

\section{CONCLUSIÓN Y DISCUSIÓN}

La producción científica en la enfermería de urgencias cardíacas y coronarias es escasa si la comparamos con otros colectivos profesionales. En este hecho pueden influir diversos factores, como son la falta de tiempo, por el horario por turnos; la falta de medios, por el impedimento al acceso a internet para los enfermeros y enfermeras desde el puesto de trabajo; la falta de formación, por el déficit en los conocimientos en materia de investigación y documentación adquiridos durante la carrera... Aun con todo, se está investigando más en 
el ámbito hospitalario, a pesar de la carga de trabajo, que en el universitario, donde los horarios son más estables y facilitaría tener más tiempo para la producción científica.

La evolución de las publicaciones en este campo durante los años analizados muestra un avance fluctuante hasta el 2008, año en el que la producción científica experimenta un claro incremento.

Los dos temas predominantes son las patologías concretas y sus tratamientos, mientras que temas relacionados con los cuidados de enfermería o los diagnósticos de enfermería son menos tratados. Serían de gran valor para nuestra profesión estudios sobre los cuidados de enfermería en el área de urgencias, de esta manera se podrían ofrecer nuestros cuidados en la dirección más adecuada para beneficio del usuario con patología cardíaca o coronaria.

Se muestra un gran interés por la investigación en países como Estados Unidos y Reino Unido, destacando de forma muy relevante, quizá por la mayor tradición investigadora que existe en estos países. No así en el resto de países europeos, de los cuales se han obtenido unos resultados meramente anecdóticos sobre su producción científica.

Un dato importante para nuestra profesión es que la mayoría de los artículos se están publicando en revistas de enfermería (ED Nursing, Journal of Emergency Nursing, Emergency Nursing, Nursing...), y la mayoría se encuentran en revistas específicas de enfermería de urgencias o enfermería cardíaca o coronaria. Ya ha sido indicada la escasez de producción científica en enfermería en este ámbito, pero si a ello le sumamos que una cuarta parte trata sobre temas médicos, dicha escasez se hace aún más evidente. Desde este artículo nos preguntamos por qué desde la enfermería no se está investigando como debería sobre sus funciones, sobre los cuidados, sobre la docencia o sobre la propia investigación en sí misma.

Más allá de la cantidad de producción científica localizada, la variedad de tipo de documentos es muy amplia. Así encontramos artículos de opinión (como cartas, editoriales) sin evidencia científica ninguna, artículos de observación (son los que más abundan), como estudios de casos, y en menor medida, estudios experimentales o de investigación (ensayos clínicos y observaciones sistemáticas) con mayor evidencia científica. Obviamente el futuro de la enfermería debería orientarse a la elaboración de documentos y potenciación de la investigación, que aporten una evidencia científica a la profesión, tal y como desde diferentes iniciativas se están elaborando desde Investen...

Los resultados obtenidos en las bases de datos son claramente negativos en lo que a la enfermería española concierne. El hecho de no haber encontrado resultados de la búsqueda en Cuiden y en el IME revela un menoscabo importante en la investigación en la enfermería de urgencias cardíacas y coronarias. Igualmente, el no haber recogido ningún resultado en las bases de datos internacionales de artículos escritos por autores españoles indica un déficit importante de la investigación en nuestro país por parte de la enfermería.

Las bases de datos son una herramienta de gran utilidad para la investigación. Pero al realizar este estudio se han encontrado ciertas deficiencias a la hora de la recuperación. El hecho de no mostrar información sobre el autor, sobre el tipo de artículo o sobre el tema del artículo dificulta mucho la tarea de localización de la información.

Puede existir un sesgo en esta investigación en la recuperación de la producción científica en las bases de datos nacionales. La falta de herramientas de búsqueda de información, hace que la recopilación de los documentos no sea tan exacta como en las bases de datos 
que cuentan con un tesauro, y que toda la información está clasificada de acuerdo a ese tesauro.

El interés por la investigación en enfermería ha de empezar ya en el ámbito universitario. Según un estudio realizado por Serrano Sastre el enseñar a partir del enfoque de la investigación estimula a los alumnos hacia la misma (7). Si los alumnos se van familiarizando con la literatura científica mientras se forman, no van a tener las mismas dificultades a la hora de realizar un estudio científico. Actualmente no hay una formación suficiente en las carreras sobre esta función de la enfermería. Es necesario que el nuevo plan de estudio, más orientado hacia la investigación, y el acceso a estudios de posgrado, potencie las deficiencias hasta ahora existentes para acceder a la investigación como para producirla.

En cuanto a las perspectivas de futuro, con la adquisición de formación en materia de investigación, se espera que los enfermeros y enfermeras se impliquen más en la investigación sobre la asistencia y los cuidados, en la investigación sobre la docencia, y en la investigación sobre la propia investigación. De esta manera se conseguirá dar a la profesión el último impulso a la ciencia enfermera.

\section{BIBLIOGRAFÍA}

1.- POTTER P, Griffin PA. Fundamentos de Enfermería Vol.I. 5aㅡ Ed. Madrid. Elsevier. 2002.

2.- Díaz Benavente M, Martín Leal C, Jiménez Aguado JM., Maya Rincón B. Producción científica de la enfermería en los hospitales de tercer nivel. Enferm Clín. 2004;14(5):257-62.

3.- Palucci Marziale MH, Costa Mendes IA, Bernadete Malerbo M. Desafíos en la divulgación del conocimiento científico de Enfermería producido en Brasil. Index Enferm. 2004; 13(47): 75-78.

4.- Jiménez Hernández JA, Ayuso García MD, Murillo Murillo R, Guillén Ríos J F. Evolución de las publicaciones periódicas españolas de enfermería: Análisis cuantitativo. Index Enferm. 2007; 16(56): 73-78.

5.- Alpi KM. Mapping the literature of emergency nursing. J Med Libr Assoc. 2006;94(2 Suppl):E107-13.

6.- Navalpotro Pascual S, Navalpotro Pascual JM. Un desafío para la enfermería de urgencias: investigar para actuar. Emergencias. 2001; 13(4): 271-278.

7.- Serrano Sastre R, Narvaiza Solis MJ.Investigar desde la teoría y la práctica. Rev Enferm. 2000 Mar; 23(3):184-91.

ISSN 1695-6141

๑ COPYRIGHT Servicio de Publicaciones - Universidad de Murcia 